\title{
Design of a Parachute Canopy Instrumentation Platform
}

\author{
Wahab M. Alshahin*, Jared S. Daum ${ }^{\dagger}$, \\ James J. Holley ${ }^{\ddagger}$, Douglas A. Litteken ${ }^{\S}$, Michael T. Vandewalleף \\ National Aeronautics and Space Administration, Johnson Space Center, Houston, TX 77058
}

\begin{abstract}
This paper discusses the current technology available to design and develop a reliable and compact instrumentation platform for parachute system data collection and command actuation. Wireless communication with a parachute canopy will be an advancement to the state of the art of parachute design, development, and testing. Embedded instrumentation of the parachute canopy will provide reefing line tension, skirt position data, parachute health monitoring, and other telemetry, further validating computer models and giving engineering insight into parachute dynamics for both Earth and Mars entry that is currently unavailable. This will allow for more robust designs which are more optimally designed in terms of structural loading, less susceptible to adverse dynamics, and may eventually pave the way to currently unattainable advanced concepts of operations. The development of this technology has dual use potential for a variety of other applications including inflatable habitats, aerodynamic decelerators, heat shields, and other high stress environments.
\end{abstract}

\section{Overview}

Entry, descent, and landing capabilities, including parachutes, lie on the critical path to human and robotic exploration of Mars. To date, the capability to actively communicate with a parachute canopy does not exist and in turn, data regarding the dynamics and health of the parachute system is unavailable. Since wired instrumentation capabilities are limited in the operational environment, wireless communication with a parachute canopy will be an advancement to the state of the art of parachute design, development, and testing. Embedded instrumentation of the parachute canopy will provide flight data within the operational environment; in turn offering engineering insight into parachute dynamics for both Earth and Mars entry that is currently unavailable. This will allow for more robust designs which are optimally designed in terms of structural loading, less susceptible to adverse dynamics such as pendulum motion and gliding, and will eventually open the door to advanced concepts of operations (CONOPS).

The Parachute Canopy Instrumentation Platform (PCIP) is a technology, currently in development, that will allow for two-way wireless communication with the parachute canopy as well as the various structural components (risers, suspension lines, etc.). This system will need to be structurally reliable in order to withstand the harsh operational environment of parachute deployment (large packing pressures, snatch loading, electromagnetic discharge) as well as ensure a clear and safe communication link between the parachute canopy and the vehicle which will not negatively affect other onboard systems.

The PCIP system is a platform that would provide a platform for implementation of a wide range of parachute canopy sensors and actuators; assuming the measurement device can be designed small and light enough as to not introduce a point mass which will impart unacceptable forces on the parachute canopy. Establishing a plug and play platform with a reliable wireless communication link would allow for a consistent method for obtaining various measurements almost anywhere on the parachute system structure. Due to the variation in timescales of dynamics of parachute operation (very quick deployments to very slow steady state descent), the PCIP system must be capable of responding quickly and be highly sensitive to measurement changes, yet possibly maintain an operational state throughout the flight of the parachute.

\footnotetext{
* GN\&C Engineer, Aeroscience and Flight Mechanics Division, wahab.m. al shahin@nasa.gov

$\dagger$ Parachute Systems Engineer, Aeroscience and Flight Mechanics Division, jared. daum@nasa.gov

${ }^{\ddagger}$ Electronics Engineer, Software, Robotics, and Simulation Division, james . j . hol ley@nasa.gov

$\S$ Structural Engineer, Structural Engineering Division, douglas. litteken@nasa.gov

ฯ Avionics Engineer, Aircraft Operations Division, mi chael.vandewal le@nasa.gov
} 


\section{A. Potential Applications of PCIP}

Once developed, PCIP may be used to provide operational measurements as a means of monitoring the parachute structural dynamics and aerodynamics. Examples of these measurements include:

- Tension in the reefing line

- Tension in the suspension lines

- Spatial canopy position at various points

- Overall parachute motion and deformation geometry

- Snatch loads seen by the payload

- Broadcloth strain

- Flow fields surrounding parachute operation (near wake, internal canopy flows, boundary layer profiles)

An example of an application of PCIP, outside of the reefing line tension measurement, is the measurement of individual riser loads for the NASA CPAS program to support the effort to quantify the effects of lead/lag seen during canopy inflation. Current data systems (button cells at riser attach point and large "dog bone" load cells wired to the payload) have failed to reliably provide riser loads. The button cell method uses load cells outside of their intended use and has provided unreliable data. The wired load cells are intrusive (place in-line with the riser) and the wiring is prone to damage during deployment and use. PCIP can provide a solution that aligns with its intended use, is non-intrusive (is added to the riser after installation on the test vehicle), and is not prone to damage from tethered wire use.

Another application of the platform is wireless disreefing cutter commanding. A two-way communication with the parachute canopy will enable the payload to send a command to an electronic cutter based on any flight software algorithm, and can significantly minimize the lead/lag associated with the tolerancing of reefing cutters. Additionally, the ability to command reefing schedules on the fly will allow for advanced concepts of operations. For example, the NASA Orion flight software and parachute system must account for many scenarios including pad abort, ascent abort, and nominal entry. Inflexibility in the reefing schedule, due to the use of fuse-based cutters, leads to a very rough discretization of the transition between phases and parachute CONOPS, resulting in sub-optimal loads and stability. For example, Orion ascent aborts resulting in parachute sequence initiation between the altitudes currently rely on deploying reefed drogues until 4000 feet, at which point the main parachutes are deployed. The drogue parachutes will not reach a full open state due to long reefing times, and the instability of the capsule and reefed drogues may cause adverse main deploy conditions. If the first and second stage reefing timers can be commanded fired quickly after inflation, the drogues can provide stabilizing forces at full open before the transition to mains. In the figure below, approximately 900 simulations of ascent aborts between the altitudes of approximately 6000 and 8000 feet, using the FAST simulation, show 397 failures due to adverse CM attitude at main deploy when using the current reefing schedule. Implementing the capabilities of on-demand reefing cutter actuation reduces these violations to 17 , greatly increasing the systems reliability. 


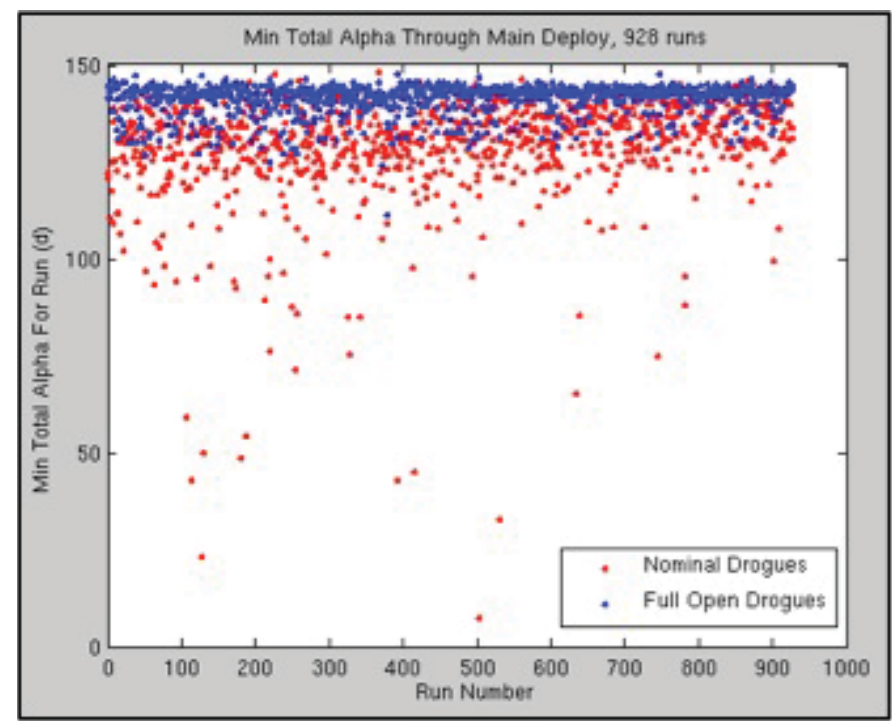

Figure 1. Benefit of on-demand disreef capability (PLOT NEEDS TO BE AIAA'd)

The ability to gather this data will prove vital for the validation of mathematical and computer models of computational fluid dynamics (CFD) and fluid structure interaction (FSI). These types of models currently exist but lack of inflight parachute data, required for validation, limits application towards design decisions. Instead, design decisions come mainly from costly and time consuming testing of parachute systems in wind tunnels and operational drop tests. Additional applications of the PCIP technology include inflatable habitats, inflatable decelerators and thermal protection systems, and conceivably any parachute system ranging from recreational use by skydivers to atmospheric entry of spacecraft vehicles.

\section{B. Major Design Considerations and Constraints}

These are the major design considerations.

Table 1. PCIP Design Considerations and Operational Constraints

\begin{tabular}{ll}
\hline \hline \multicolumn{1}{c}{ Design Consideration } & \multicolumn{1}{c}{ Description and Rationale } \\
\hline $\begin{array}{l}\text { System size } \\
\text { System mass }\end{array}$ & Minimally intrusive or nonintrusive measurement and communication hardware \\
Measurement accuracy & Minimized to not impact the safe and effective operation of the parachute \\
Signal encryption & Reliably collect data through high rate dynamics \\
Power requirements & Depending on application, determine if encryption is required \\
Usage cycles & Minimize power to reduce required size and mass \\
\hline \multicolumn{1}{c}{ Operational Constraints } & Optimize design to meet desired lifespan of each system \\
\hline Packing Pressure & \multicolumn{1}{c}{ Description and Rationale } \\
Temperature & System must withstand packing compression force if applicable \\
Atmospheric Pressure & System must withstand potentially large changes in temperature \\
Humidity & System must withstand potentially large changes in pressure \\
Shock and Vibration & System must withstand potentially large changes in humidity \\
Electromagnetic interference & Parachute operation is violent, electronics must be durable \\
Radiation & Survive and operate under the EMI environment \\
\hline \hline
\end{tabular}


This paper will outline the requirements and design considerations that are necessary in order to design the PCIP system, bench test it in a controlled environment, and eventually test it through an operational flight test.

\section{Electronics Overview}

Many challenges in developing PCIP will be seen in the development of the electronic hardware and packaging. A lightweight, modular and robust electronic solution will be required to instrument the canopy. Systems must tolerate large pressures during parachute packing, then withstand large load transients as the parachute deploys and inflates. Furthermore, to prevent snatch loading during parachute deployment induced by integrated electronics, the density of the solution must be less than the material in its vicinity. A modular solution that can support a suite of sensors is preferred, as several variables, including canopy position, motion, fabric strain, opening force, air velocity and air pressure will need to be measured. Commands may also be sent to the system, allowing real time control of reefing cutters, which would require more complex digital circuitry or a microcontroller. Technologies in development that could provide such a solution are flexible PCBs and conductive fabric. Flexible PCBs could be integrated directly into the parachute fabric, providing sensing at precise locations while maintaining a low mass, low density solution. Several manufacturers produce conductive fabrics that can be used as EMI shielding. This would provide a lightweight, soft goods EMI protection that could also be integrated directly into the parachute fabric. A study of sensor packages, chips, microcontrollers, and wireless communications systems will need to be conducted in order to understand the operating capabilities and ease of integration into a modular solution. Modification or customization may be necessary for packaging the sensors into the parachute.

In order to capture the relevant data from sensors located in a parachute canopy, it is desired that the electronics be capable of collecting data at a $1 \mathrm{kHz}$ bandwidth. This bandwidth is also sufficient to serve as a control loop possibly actively actuating some portion of the parachute canopy. The electronics will also need to interface with a radio module in order to send and receive commands from the payload.

\section{A. Control and Sensing}

Several commercial off the shelf microcontrollers and analog to digital conversion products exist that satisfy the requirements of PCIP. A microcontroller, possibly with built in analog to digital converters could be selected to achieve the requirements. Additional considerations when selecting this component should be sufficient outputs for interfacing with the radio module and sensors. Common interfaces and ports on the microcontroller are also desired to increase the modularity of the package. The microcontroller should be selected to consume as low power as possible, reserving power for sensors and radio communications.

\section{B. Power}

It is desired that data logged at $1 \mathrm{kHz}$ be sampled for 20 seconds to 1 minute. PCIP is wireless, so for initial implementation, batteries will have to be used. Because the length of time samples will be taken is relatively short, battery selection should have a range of possibilities. Battery selection also needs to take into consideration the possible excitation voltages of the analog sensors. A modular battery implementation allowing for several options of voltage level to accommodate for different sensor packages should be considered.

\section{Shielding}

One of the major considerations for the design of the electronics is ESD (electrostatic discharge) protection. Friction between cloth in the parachute as it is deployed will cause electrostatic charge to build up temporarily before it is discharged into the surrounding air. This charge build up is currently estimated at $24 \mathrm{kV}$ (GET CHART FROM EV GUYS), which exceeds the highest commercial standards $(15 \mathrm{kV})$. This requires that a custom implementation be designed to meet these requirements. Packaging of the electronics will also have to be shielded - preventing ESD from affecting the electronics except at protected locations along the interfaces of the package.

\section{Packaging}

Electronics will have to be packaged such that they are not powered on during stowage and able to survive packing pressures, designed to prevent tearing and damage to the parachute, and able to activate the electronics upon 
deployment. Packing pressures for different parachutes will have to be withstood (pull table from packing pressure research).Designing the packaging to mimic reefing line cutters - hardware that has previously been used in parachute canopies - is most desirable since they have already been proven to survive all of the requirements, as well as activation upon deployment.

\section{Wireless Communication Design Considerations}

Radio Frequency (RF) communication integration will be important to the implementation of PCIP. There are a number of important and unique design considerations relative to similar ground-based RF implementations. First and foremost, the system must be able to tolerate the high electrostatic fields inherent in atmospheric re-entry (including parachute deployment and descent). Characterizing this environment is the first step in determining the expected noise distribution that the system will encounter. The link margin analysis, determined by several important design considerations, will then follow. As a starting point, the required number of channels, sensor bandwidth, and transmission rate/schedule will set the minimum data throughput requirements, which will then be contrasted with the noise environment. This comparison between data requirements and the noise environment, along with the required safety margins, will constrain the modulation type, symbol rate, link protection/encryption scheme (to prevent unauthorized activation), error correction/detection, power requirements, and antenna gains for the system.

Two main wireless functions are desired of the PCIP system:

1. Transmission of strain data from parachute electronics package(s) to payload electronics package in real-time.

2. Transmission of commands from payload electronics package to parachute electronics package, such as a parachute disreef command.

\section{A. Frequency Band Selection}

Frequency band selection is the first priority in designing a wireless system within the given project constraints. For a project with minimal available budget, COTS equipment must be used to the greatest extent possible, while still meeting the project requirements. With such a wide pool of available, proven, and low-cost devices in the $915 \mathrm{MHz}$, $2.45 \mathrm{GHz}$, and $5.8 \mathrm{GHz}$ Industrial, Scientific, and Medical (ISM) bands, choice of one of these bands is attractive assuming they can support the required data rates within the power/bandwidth constraints imposed by law. Even if special authorization is approved for lifting these constraints in our application, in doing so the design work and cost increases dramatically. Lastly, power levels for these devices are reasonable given the limitations of current battery technology.

In selecting an appropriate frequency band in a power and size-constrained application (such as a parachute canopy), it is necessary to evaluate the benefit of minimizing the antenna size relative to increasing the path loss. In other words, for a higher frequency system to maintain the same link margin as a lower frequency system, the transmit power or antenna gains must be increased to cover the increased path loss. With very strict mechanical installation constraints, such as the reefing line of a parachute canopy or the top of a spacecraft capsule, these considerations are very important higher-gain antennas occupy a significantly higher volume.

\section{B. Transceiver Selection}

As a part of the frequency selection process, a survey of commercially available transceivers is necessary. Due to wide use of the ISM bands in commercial products, there are many transceiver modules available on the market today that are under \$10 USD. The following aspects of a transceiver need to be addressed in the final selection:

- Agile oscillator for spread spectrum operation

- Frequency-hopping Spread Spectrum (FHSS) - Blanking interval

- Direct Sequence Spread Spectrum (DSSS) - Aquisition time

- Data rate selection/programmability

- Receiver sensitivity at a given data rate and modulation type

- Transmit power maximum, minimum, and step programmability

- Component count and complexity of supporting electronics design

- Balanced/unbalanced antenna interface 
- Transmit/receive switching

$\circ$ Filtering

- Built-in packet handling

○ Preamble/sync detection

- Addressing

- Symbol decoding

- Forward error correction

- Data integrity checks (such as Cyclic Redundancy Check)

- Encryption for command link protection

- Transmitter power consumption

- Low-power sleep modes

○ Allows battery to power transceiver for long periods of time while waiting for start command

Once candidate transceivers are eliminated for lack of required features, the remaining transceivers should be tested using an RF attenuator to simulate the loss conditions considered in the next section. Efficient packet construction and careful selection of error correcting and detecting codes is critical to a workable final system. Packet error rate tests may be easily performed using development boards provided by the transceiver manufacturers this type of product is nearly universally available from manufacturers of these ICs.

\section{Antenna Selection}

The proper antenna system design is critical, as it is in all wireless systems. Once a band is chosen, there are two antenna selections to make one for the parachute electronics and one for the payload electronics. The parachute electronics antenna is the most size and weight constrained of the two, given that it will be installed within or around the parachute material and must not interfere with its aerodynamic characteristics. Due to the geometry of potential installation sites (reefing line, risers, etc.), there are only a limited number of practical (small, durable, simple) antenna designs for this application. The combined gain of the parachute and payload antennas at the worst-case sway angles must be considered in the final link margin analysis. Additionally, cross-polarization losses should be minimized by careful antenna selection, as twisting along the axis of travel is an unavoidable reality.

\section{Electrostatic Discharge Susceptibility}

Triboelectric charging of the parachute material occurs due to friction in the parachute fabric, as parachutes are often packed into small containers at immense pressures. This results in a charge buildup, followed by an air discharge if the buildup is sufficiently high.

This is a significant risk given that the maximum field strength at the parachute and at the payload are unknown at this point in time. In order to mitigate this risk, testing should be performed with the goal of accurately estimating the peak field strength generated by the opening of the parachute canopy. Once the upper limit has been established, prototypes can be tested using standard test procedures. In any case, this limit should be established before detailed design of the system in order to minimize the cost/schedule impact of redesign.

Once survivability is ensured, it is important to delay data transmission until the electrostatic effects have subsided. This delay could most easily be implemented by delaying transmission in software for a fixed amount of time after initial activation. Additionally, adaptive sensing in the parachute or payload electronics could determine a proper activation time based on the environment. Either way, this will have to be given consideration in the final design.

\section{Structural Design Considerations and Instrumentation}

\section{A. Parachutes}

A basic parachute structure is made up of a series of high strength materials connected to a drag-producing canopy. Generally, multiple components and materials are the load carrying members of the structure that transfer the loads generated from the canopy to the payload. To measure these loads during flight, the various components must instrumented, using an appropriate strain measurement device. Because of this variation throughout the structure, the gauge device that is used should be selected specifically for that type of line and loading condition, as device performance 
will vary among sensors. PCIP is currently developing the capabilities to examine three main lines in the parachute: the risers, the suspension lines, and the reefing line. The risers are made of a bundle of cords that link the payload to the suspension lines. The suspension lines consist of a series of single cords that connect the riser to the canopy. The reefing line is used to reef the canopy during parachute inflation and is made of a single cord looped around the hemisphere of the skirt of the parachute.

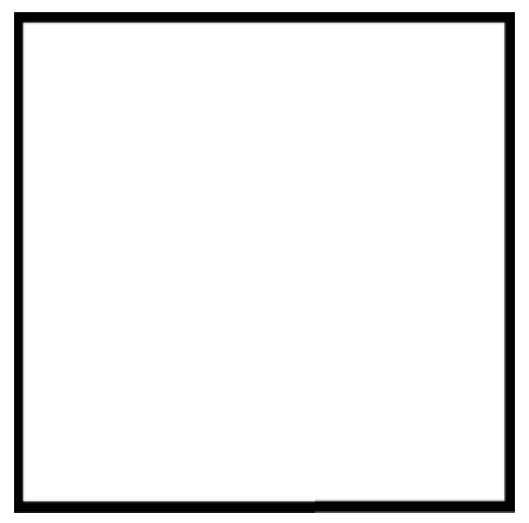

Figure 2. Picture of Parachute Structure or Reference Picture

Measuring strain and load in a structure provides valuable data for the further design and development of the system. For parachutes especially, understanding of the actual loads transferred during a drop test will help in building an efficient and lightweight structure. Traditional structural strain monitoring with metallic materials uses foil strain gages applied directly to the surface of the metal that measure the linear behavior of the material. Lightweight structures and parachutes, however, are made of high strength textile materials that behave in both a linear and non-linear fashion and exhibit high strain fields that exceed the strain capacities of foil gages. Direct measurements of high-strain fabrics have traditionally been done at NASA using optical methods that involve expensive cameras, lights, and surface preparation. ${ }^{7}$ This type of measurement is very accurate, but only beneficial for ground tests and not applicable for flight tests or parachute drop tests. In line load measurements of parachutes has also been used in the past, but involve heavy duty load cells and additional hardware that can only be implemented in specific parts of the system, specifically on the riser near the attach point. A direct strain measuring device that is flexible and capable of being integrated into the textile structure is necessary to accurately monitor strain in a lightweight vehicle, or parachute.

To adequately measure high-elongation loads of parachute lines, one must consider the following characteristics in choosing a gauge device: the ability to measure and stretch to high strains (10-50\%), the capability of withstanding and measuring peak loads during a dynamic loading event, the ability to be ruggedly adhered to or integrated with a textile line and staying fixed during the entire inflation sequence, and the ability to be integrated with the textile line such that it can be packaged and limit snag hazards or interference with other components. Identified here are a variety of potential gauge devices for this application. These devices work using resistive properties where a base material is conductive and has a natural internal resistance. When the material is stretched, the resistance changes and that change in resistance can be correlated to a change in stretch and strain. To create a measuring device from these materials, an excitation voltage is input and the change in output voltage is measured over the resistive material using a Wheatstone bridge circuit. A traditional foil strain gauge, typically used for metals, utilizes the same principal. ${ }^{4}$

\section{B. Instrumentation Options}

Two conductive materials are currently being evaluated for the PCIP gauge device. The first is a conductive polymer stretch sensor made by Images SI, Inc. This material is made in a small diameter cord, similar to a rubber band, and is mounted directly to the surface of a textile. The material has been used on robotics and as wearable sensors, for detecting breathing patterns, for example..$^{5}$ Small scale testing has shown high-strain capabilities and high data rates on dynamic events. The other material we are evaluating is a conductive Metal Rubber ${ }^{\mathrm{TM}}$ by NanoSonic, Inc. This unique material is a self-assembled free-standing electrically conductive elastomer that can be used on a high stretch substrate or as a coating on other materials. ${ }^{6}$ The Metal Rubber ${ }^{\mathrm{TM}}$ material can be cut into sensor shapes and has 
been used in multiple applications for structural strain sensing, including on high-tension Kevlar straps of NASAs Hypersonic Inflatable Aerodynamic Decelerator (HIAD), ${ }^{1}$ and on small diameter Zilon cords on NASAs Ultra-Long Duration Scientific Balloons. ${ }^{3}$ These tests have shown that the Metal Rubber sensor has high-strain capabilities and a unique ability to be sized for a specific application and loading regime. We are also evaluating a third strain gauge device that uses traditionally instrumented pins in a fixture that attaches to the cord to measure the load. This device, known as a seat belt tension device, is made by Novatech Measurements, Ltd and has also been used successful on a flight test of NASAs HIAD vehicle. The HIAD testing of these devices has shown excellent high-strain capabilities, high accuracy, and highly reliant systems throughout the course of both ground and flight testing. ${ }^{8}$

Based on parachute loading profiles, we are most interested in the gauge performance under dynamic loading and constant tension, representing the deployment and inflation of the parachute. Each device will be tested on a benchtop load frame with single cord level representative loads in tension. Dynamic loading will be tested with small scale drop tests of the expected deployment loads of a single cord. Packaging and stagnation tests will also be completed to characterize the impact of pre-test parachute packing on the gauge and its measurement capability. The results of these tests will be used to compare and select the primary gauge device used for small scale and full scale testing of the entire PCIP system.

\section{Testing}

Testing of the PCIP system will consist of environmental, bench, ground, and flight testing, resulting in a technology demonstration in Fall 2015. Data has been gathered at the NFAC 80x120 wind tunnel at Ames Research Center regarding the EMI environment generated by scaled NASA Capsule Parachute Assembly System (CPAS) main parachute operating at steady state conditions. Further data will be collected using established test techniques at KSC to define the maximum EMI environment during deployment and inflation. Bench and ground testing of the electronics and RF system will fully characterize the antenna patterns and performance characteristics of the system. Bungee tests and other indoor drop tests will demonstrate the systems survivability under harsh deployment conditions and will test the accuracy and capability of the measurement system under stressing conditions. Flight tests are anticipated using existing hardware and test techniques, which will have been fully developed and demonstrated Spring 2015. The goal is a technology demonstration on a CPAS full-scale drop test, providing individual riser loads and/or reefing line tension, in addition to potentially demonstrating the ability to wireless command actuation of an electronic reefing cutter.

\section{Conclusion}

Beginning with the definition of the operational environment and packaging and performance requirements, a survey of components to be integrated in the PCIP system is conducted. This includes state of the art technologies in the fields of wireless communication, structures, flexible materials, and lightweight electronics. The design and testing roadmap is defined, culminating in a full scale test in the operational environment on a NASA CPAS drop

test. The benefits of the capabilities provided by PCIP are thoroughly discussed, including validation of computer models, reduction of safety margins, advanced concepts of operations through wireless operation of canopy actuators, and greater knowledge of parachute performance, which can be used in the design cycle of any parachute.

\section{References}

[1] Cassell, A. M., Swanson, G. T., Quach, B. T., Kushner, L. K., Brown, J. D., Kazemba, C. D., and Cheatwood, F. M. (2013). Design and Execution of the Hypersonic Inflatable Aerodynamic Decelerator Large-Article Wind Tunnel Experiment. AIAA Aerodynamic Decelerator Systems (ADS) Conference. Daytona Beach, Florida.

[2] Gioberto, G., and Dunne, L. (2012). Theory and Characterization of a Top-Thread Coverstitch Stretch Sensor. IEEE International Conference on Systems, Man, and Cybernetics, (pp. 3275-3280). Seoul, Korea.

[3] Hill, A. (2014, January). Low-Weight, Durable, Low-Cost Metal Rubber Sensor System for Ultra-Long-Duration Scientific Balloons. NASA Tech Briefs, pp. 22-23.

[4] Hoffmann, K. (2001). Applying the Wheatstone Bridge Circuit. Darmstadt, Germany: HBM Publication. 
[5] Khor, J. M., Tizzard, A., Demosthenous, A., and Bayford, R. (2014). Wearable sensors for patient-specific boundary shape estimation to improve the forward model for electrical impedance tomography (EIT) of neonatal lung function. Physiological Measurement.

[6] Lalli, J. H., Claus, R. O., Hill, A. B., Mecham, J. B., Davis, B. A., Subrahmanyan, S., and Goff, R. M. (2005). Commercial Applications of Metal Rubber. Proceedings of SPIE.

[7] Litteken, D., Selig, M., Valle, G., and Oliveras, O. (2012). Utilizing Photogrammetry and Strain Gage Measurement to Characterize Pressurization of an Inflatable Module. AIAA Structures, Structural Dynamics, and Materials Conference. Honolulu, Hawaii.

[8] Swanson, G. T., Cassell, A. M., Johnson, R. K., Hughes, S. J., Calomino, A. M., and Cheatwood, F. M. (2013). Structural Strap Tension Measurements of a 6 meter Hypersonic Inflatable Aerodynamic Decelerator under Static and Dynamic Loading. AIAA Aerodynamic Decelerator Systems (ADS) Conference. Daytona Beach, Florida. 